\title{
Detection of Hydrocephalus Lateral Ventricles Quantitatively in Brain MRI images of Infants
}

\author{
S. Javeed Hussain \\ Department of ECE, \\ BCETFW, Kadapa. \\ Andhra Pradesh
}

\author{
P.V. Sree Devi, Ph.D \\ Department of ECE, \\ AU, Vishakapatnam, \\ A.P, India.
}

\author{
T. Satya Savithri, Ph.D \\ Department of ECE, \\ JNTUH, Hyderabad. \\ A.P, India
}

\begin{abstract}
In this paper, a new clustering method is applied to segmentation and volume change assessment in lateral ventricles affected by hydrocephalus pathology in the infants. The hydrocephalus is characterized as such, which results from excessive accumulation of cerebrospinal fluid in the ventricles, leading to enlargement of cerebral ventricles. The complex shape o the ventricular system is evaluated by a visual assessment of MRI scans, but still there is necessity to note the amount of change in volume. We present a algorithm with adjustable feature weight with Optimal feature selection using validity function. The algorithm is called Optomal feature Weight adjustable FCM with Gaussian smoothing (OfwaFCM). There was need to develop new algorithm for segmenting non-symmetric images. The objective of this algorithm is to produce fine clustering results and to reduce effect of noise. In this method, clustering centroids and functions are used to evaluate the clustering results and validity fuction based on fuzzy partitioning is used to select Optimal Feature Weights. The results produced states that the proposed scheme provides Volume calculation and clustering performances for Detecting and segmenting the hydrocephalus.
\end{abstract}

\section{Keywords:}

Feature Weighted Vectors, Gaussian mixture model, Fuzzy cmeans, Fuzzy medical image processing

\section{INTRODUCTION}

Hydrocephalus is resulted by neurological disorder, which involves in an abnormal accumulation of cerebrospinal fluid in the brain cavities stated as cerebral ventricles. The cerebral ventricles and subarachnoid space in the brain is surrounded by the cerebrospinal fluid. The damage of brain tissues and several brain malfunctions can be result, with increased pressure on the brain by the fluid and causes ventricle enlargement to state simply about hydrocephalus is that resulted from excessive accumulation of cerebrospinal fluid. The complex shape of the ventricles commonly can be evaluated by observing the differences in the angulations of slices combined with slight differences in the positioning of the head from MRI study to the next image. This makes the assessment of the volume change calculation is desirable quantitatively. The figure 1 shows descriptive representations of the fluid pathway from ventricles to the subarachnoid space in a sagittal plane (left) and through the encountered spaces as proposed in [1] (right).

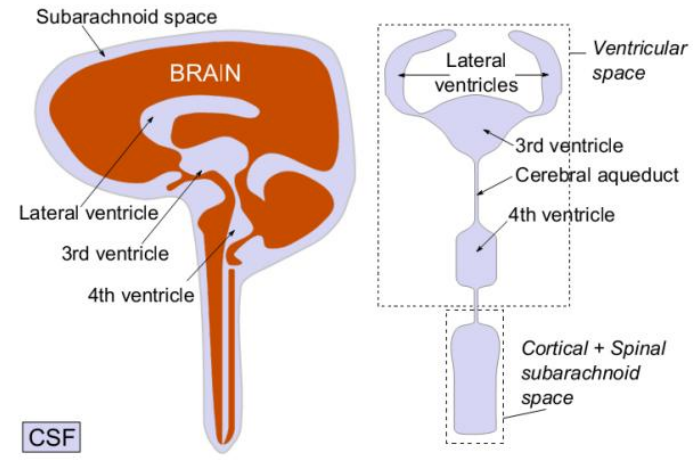

Figure 1: The cerebrospinal Fluid circulates from ventricles where it is produced to the subarachnoid space where it is resorbed (left). The Fluid flows through the various encountered spaces from the ventricles to the cortical subarachnoid space as proposed in [2] (right).

Hydrocephalus is classified into two as communicating and non-communicating depending upon the fluid pathway is obstructed or not [2]. To diagnose hydrocephalus based on imaging techniques such as computed tomography(CT), Magnetic resonance imaging (MRI).

In routine clinical practice, a ventricle enlargement can be identified by a magnetic resonance imaging sequence, while detecting the fluid flow malfunction may require an additional cine phase-contrast magnetic resonance imaging sequences.

The quantitative assessment of ventricular volume in earlier methods are stated, diagonal ventricular dimension[3], the frontal and oceiptal horn ratio[4], the ventricular brain ration[5], the Evans ratio [6], Huckman's measurement [7,8] and minimal lateral ventricular width [9]. The previous attempts to assess the volume of ventricles was focused mainly on ratio, linearity, (or) surface area estimates of ventricular size. The previous techniques were time consuming in assessing volumetric changes 3 -dimensionally [10]

\section{PROPOSED METHOD}

This paper describes a novel method to segment and to measure the volume of the ventricles using MRI scans. Initially in this method the segmentation of cerebral ventricles is performed by weighted features FCM with Gaussian smoothing scheme (WFGFCM) and in the final stage the volume of ventricles is calculated and evaluated.

\subsection{Optimal Feature Weight adjustable Fuzzy C-means Using Gaussian smoothing (OfwaFCM):}

The 2-D circularly symmetric Gaussian convolution operator is used to remove details, noise and to blur or smoothen the 
image. The symmetric Gaussian is represented in equation (1).

$$
\begin{aligned}
& G_{s}(x, y)=1 / 2 \pi \sigma^{2} \cdot e^{z} \\
& \text { Where } z=x^{2}+y^{2} / 2 \sigma^{2}
\end{aligned}
$$

The edges and smoothing is done in a better form as compared than similarly sized mean filter. The Gaussian function outputs a 'weighted average' of each pixel neighborhood, this will leads towards the value of the central pixel. The standard FCM aim is to find the centroids and to minimize the dissimilarity function. The membership matrix $(\mathrm{U})$ is initialized according to equation (2).

$$
\mathrm{M}=\sum \mathrm{Uij}=1, \quad \mathrm{j}=1, \ldots \mathrm{n}
$$

The algorithm is an iterative optimization that minimizes the dissimilarity (cost) function defined in equation (3)

$$
\begin{aligned}
& \mathrm{J}\left(\mathrm{u}, \mathrm{c}^{1}, \mathrm{c}^{2} \ldots . \mathrm{c}^{\mathrm{n}}\right)=\sum \mathrm{j}_{\mathrm{i}} \\
& \sum \mathrm{j}_{\mathrm{i}=}=\Sigma \mathrm{Uij}^{\mathrm{k}}{ }_{\mathrm{Dij}}{ }^{2}, \quad 1 \leq \mathrm{K} \leq \mathrm{D} ;
\end{aligned}
$$

Where $\mathrm{Ci}$ is the centroid of cluster $\mathrm{i}$;

$\mathrm{D}_{\mathrm{ij}}$ is the distance measure between ith centroid (ci) and jth data point.

$\mathrm{M}$ is the membership function between 0 and 1 ;

$\mathrm{K} €[1, \mathrm{~d}]$ is a weighting exponent

The distance measure is the distance of data $\mathrm{x}_{\mathrm{jm}}$ from cluster of $\mathrm{i}^{\text {th }}$ cluster, Vim in the $\mathrm{m}$ th dimension.

$$
\left.\mathrm{D}_{\mathrm{IJ}}=\sqrt{\sum(X j m}-\operatorname{Vim}\right) 2
$$

To adjust the weighting of each input feature, equation (3) can be modified $b$ adding factor which because.

$$
\left.\mathrm{DIJ}=\sqrt{\sum(\alpha} X J M-V I M\right)
$$

The greater narration in some features of the date provides important information. Standard deviation is a usual measure of variability of random sample, defined in equation (6)

$$
\mathrm{SD}=\sqrt{\varepsilon(x j-x)^{2}} / \mathrm{n}-1
$$

Where $x=1 / n \Sigma x j$

The coefficient of variation which is independent is defined by

$$
\mathrm{Cv}=\mathrm{s} / \mathrm{x}
$$

For a random samples representing $\mathrm{jth}$ sample for $\mathrm{j}=1, \ldots . . \mathrm{n}$ then the coefficient of variation of $\mathrm{k}^{\text {th }}$ feature is defined in equation(7).

$$
\mathrm{CV}=\mathrm{S}_{\mathrm{K}} / \mathrm{x}_{\mathrm{K}}
$$

$$
\begin{aligned}
& \text { Where } \mathrm{S}_{\mathrm{k}}=\sqrt{\varepsilon(x j k-x k)^{2}} / \mathrm{n}-1 \\
& \mathrm{x}_{\mathrm{k}}=1 / \mathrm{n} \sum \mathrm{x}_{\mathrm{jk}}, \mathrm{k}=1, \ldots \ldots \ldots \ldots \infty
\end{aligned}
$$

The kth feature weight can be defined as expectation of normalized $\mathrm{cv}_{\mathrm{k}}$.

$$
\mathrm{W}_{\mathrm{k}}=\mathrm{E}\left(\mathrm{W}_{\mathrm{k}}\right)=\int w d f k(w)
$$

From the calculation of the sample mean of $\mathrm{W}_{k}$ for all $b$ number of replication, the estimated feature weight $\mathrm{W}_{\mathrm{k}}$ us found as in equation(10).

$$
\mathrm{Wk}=\frac{1}{B} \sum \mathrm{W}_{\mathrm{k}}(\mathrm{b})
$$

The FCM need $s$ to be fine tuned to be insensitive to noise in most cases, there is only input feature available for clustering. Then the feature weights calculated is applied to the FCM with adjustable feature weights factors as WFGFCM, for fine tuning and to obtain required clustering results.

\subsection{Proposed Algorithm:}

Step1: convert image data I to an array of data, data 1

Step2: create 2-D Gaussian low pas filter with size and standard Deviation, sigma

$$
\mathrm{G}_{\mathrm{lpf}}=\mathrm{F}_{\mathrm{Gau}}(\text { Size,Sigma })
$$

Step3: convolve Data1, by the step3 to produce Data2:

$$
\text { Data2 }=\mathrm{G}\left(\text { Data } 1, \mathrm{G}_{\mathrm{lpf}}\right)
$$

Step4: create the input data array by combining Data1 and Data2.

Data $=[$ Data1,Data2];

Step5: find the estimated optimized feature weighing factor $\mathrm{w}_{\mathrm{k}}$ from equation (10)

Step6: apply the weighted feature factors of OfwaFCM.

Step7: this image I of array features data with feature weighing factor is applied to FCM.

Step 8: set the number of clusters $(\mathrm{C} 0$, number of maximum iterations, max in FCM.

Step9: output, set of clustered regions.

Algorithm: combining Gaussian Smoothed and original images by OfwaFCM.

\subsection{Volume Calculation of Hydrocephalus:}

After detection and segmentation of Pathology Hydrocephalus is identified. It is quite important to calculate the variation in size of the ventricles to perform the volume variation of cerebral ventricles.

To calculate the volume of the ventricles we have to initially calculate single pixel volume. The single pixel volume is described stated equation.

$$
V_{s p}=k \cdot S_{m} \cdot S_{n}
$$

The term $\mathrm{K}$ is considered as slice thickness and the $S_{m} . S_{n}$ are the spacing of pixels with respect to the dimensions. These values of the parameters can be obtained from the header of the DICOM file. The volume of the ventricles can be computed by considering the count of pixels present in the region and it has to be multiplied by the result of single pixel volume and the equation stated are used to obtain the volume and they are illustrated as 


$$
\begin{gathered}
x y \\
V_{c}=V_{s p} \sum_{\mathrm{n}=1 \mathrm{n}=1} V(m, n) \\
x y \\
V_{H}=V_{s p} \sum_{\mathrm{n}=1 \mathrm{n}=1} S(m, n)
\end{gathered}
$$

Where

$V_{c} \quad$ - Complete volume of brain

$V_{H} \quad$ - Complete volume of hydrocephalus

$x, y$ - Dimensions of single cross section in pixels

$\mathrm{V}$ - Binary image of brain

S - binary image of hydrocephalus

$\mathrm{m}, \mathrm{n}$ - coordinates of pixels

The HBR ratio is computed using the values of $V_{H}$ and $V_{C}$ as

$$
V_{H / c}=\left(\frac{V H}{V C}\right) * 100
$$

\section{EXPERIMENTAL RESULTS}

The proposed hydrocephalus segmentation and Detection is implemented in the working platform MATLAB (version7.10) and it is evaluated using brain MRI images, which are collected from diagnostic centre. The Mri studies were from patients whose outcome (normal, stable, hydrocephalus) was known and were selected by a radiologist to reflect a range of outcomes. Manual segmentation was also provided by the radiologist, so that segmentation portion of the frame work could be validated.

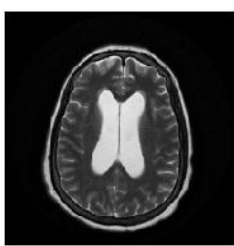

(a)T2-Weighted image

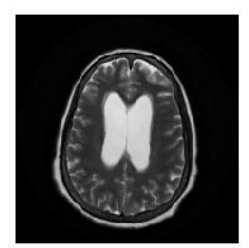

(b)T2-Weighted image

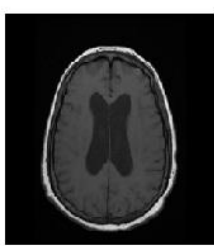

(c)T1-weighted image
Figure 2: input images of 3 patients suffering from hydrocephalus

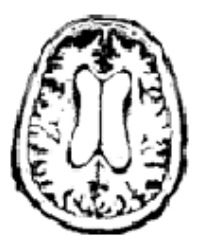

(a)

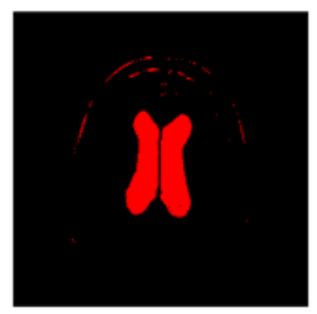

(b)

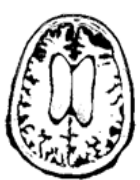

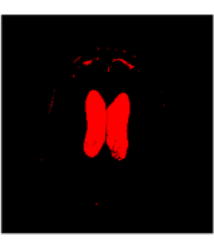

(b)

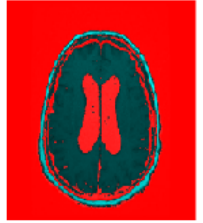

(c)
Figure 4: Segmented outputs of case $2 \& 3$ with drocephalus of T2 \& T1 weighted images

\subsection{Performance Analysis}

The frame work of segmentation of the cerebral ventricles is computed using the similarity index, $S_{I}$ between the segmented results obtained and a manual segmentation

$$
S_{I}=\left(\sum S x\right) / X
$$

The results obtained using the similar index $S_{I}$ is provided in the Table 1. It is stated that if the value of $S_{I}$ is greater than $70 \%$ it indicates the excellent agreement. This has been evaluated on 5 cases of hydrocephalus to register the agreement in the result. The mean accuracy of the result is obtained is 80.18

The Table 1 Provides the information stating the similarity accuracy found in segmentation of the hydrocephalus pathology in the infant brain.

Table 1: Evaluation of Accuracy by Similarity Index

\begin{tabular}{|l|l|}
\hline Image No's & Similarity index $\left(S_{I}\right)$ \\
\hline 1 & 78.4 \\
\hline 2 & 82.1 \\
\hline 3 & 79.5 \\
\hline 4 & 77.2 \\
\hline 5 & 83.7 \\
\hline
\end{tabular}

The Table 2 provides the information regarding the volume change of the ventricles in comparison with complete brain and the assessment change of the ventricles with normal ventricles is represented. The Volume calculation is evaluated by considering $5 \mathrm{MR}$ images and the computed values are presented as,

Table 2: Ratio of Volume Change in Lateral Ventricles

\begin{tabular}{|l|l|l|l|}
\hline $\begin{array}{l}\text { Image } \\
\text { No }\end{array}$ & $V_{c}\left\{C M^{3}\right\}$ & $V_{H}\left\{C M^{3}\right\}$ & $\begin{array}{l}\text { HBR Ratio } \\
(\%)\end{array}$ \\
\hline 1 & 1110.27 & 428.75 & 38.61 \\
\hline 2 & 1048.12 & 398.64 & 38.03 \\
\hline
\end{tabular}




\begin{tabular}{|l|l|l|l|}
\hline 3 & 998.76 & 364.76 & 36.51 \\
\hline 4 & 1101.81 & 401.72 & 36.46 \\
\hline 5 & 1045.64 & 210.11 & 20.09 \\
\hline
\end{tabular}

\section{CONCLUSION}

FCM is one of the most well-known clustering algorithms. But it is sensitive to noise and its performance is limited by the equal feature weights of the standard FCM. This method has quantitatively segmented the lateral ventricles and assessment of Volume changes is computed for the Hydrocephalus Pathology is done in brain Mri images. The segmentation method, is intensive on Gaussian smoothed image with weighting factors selection using Quantitative adjustment approach known as WFGFCM is conducted. The method was experimented on MRI images with and without added noise. Apart from observation of the clustering results in the specific application of brain ventricular compartment segmentation, results show that our proposed WFGFCM scheme is more insensitive to noise and leading to better clustering performance and detects the Hydrocephalus in the infants.

\section{REFERENCES}

[1] Rekate, H.L. A consensus on the classification of hydrocephalus: its utility in the assessment of abnormalities of cerebrospinal fluid dynamics, child's nervous system 2011;27(10):1535_1541.

[2] Matsumae, M.,Kikinis , R., Morecz, I.horenzo, A.v.,Albert, M.S.,Black, P.M.,et al. Intracranical compartment volumes in patients with enlarged ventricle assessed by magnetic resonance-based image processing. Journal of Neurosurgery 1996;84(6):972-981.
[3] A.H.Mesiwala, A.M.Avellino, and R.G.Ellenbogen," the diagonal ventricular dimension: a method for predicting suunt malfunction on the basis of changes in ventricular size,"Neurosurgery,Vol.50, no.6,pp.1246-1252,2002.

[4] B.B.O'Hayon, J.M.Drake, M.G.Ossip, S.Tule, and M.clarke, "frontal and occipital horn ratio: a linear estimate of ventricular size for multiple imaging modalities in pediatric hydrocephalus," pediatric Neuro surgery, vol.29,no.5,pp.245-249,1998.

[5] V.Synek and J.R.ruben," the ventricular brain ratio using plainmetric measurement of EMI scans," British journal of Radiology,vol. 49,no.579,pp.233-237,1976.

[6] J.Evans, the verification of computer algorithm designed to calculate the volume of the human cerebral ventricles based on CT images, B.S.Thesis, Dalhousie university, Halifas, Canada,2005.

[7] M.s.Huckman ,J.Fox and J.Topel," the validity of criteria for the evaluation of cerebral atrophy by computed tomography", radiology, vol.116,no.1,pp.85_92,1995.

[8] J.H.Fox, L.T.Jordan and M.S.Huckman," use of computed tomography in senile dementia", Journal of neurology Neurosurgery and psychiatry, vol. 38,no.10.948-953,1975.

[9] B.S.Brann iv, C.Qualls, L.Wells and L.A.papile," A symmetric growth of the lateral cerebral ventricle in infants with posthemorrhagic ventricular dilation," Journal of pediatrics,vol.118,no.1,pp.108-112,1991.

[10] R.w.Sze, V.Ghioni, E.Weinberger, K.D.Seidel, and R.Ellenbogen, "Rapid computed tomography techniques to measure ventricular volumes in the child with suspected ventriculoperitoneal stunt failure: validation of technique with a dynamic phantom", Journal of Computer Assistanced tomography,vol.27,no.5.663667,2003 . 